\title{
Estadísticas e indicadores de rendimiento de colecciones y servicios bibliotecarios de carácter electrónico: estudio de caso de las revistas electrónicas
}

\author{
Por Ángel Borrego y Cristóbal Urbano
}

Resumen: Se analiza el uso de estadísticas e indicadores de rendimiento de productos y servicios electrónicos en los procesos de evaluación bibliotecaria. Se examinan los principales proyectos de definición de estadísticas e indicadores desarrollados durante los últimos años, prestando especial atención a tres de ellos: Counter, E-metrics e ISO, y se analizan las estadísticas que actualmente ofrecen cuatro grandes editores de revistas electrónicas (American Chemical Society, Emerald, Kluwer y Wiley) y un servicio (Scitation Usage Statistics) que aglutina datos de seis editores de revistas de física. Los resultados muestran un cierto grado de consenso en la determinación de un conjunto básico de estadísticas e indicadores a pesar de la diversidad de proyectos existentes y de la heterogeneidad de datos ofrecidos por los editores.

Palabras clave: Estadísticas, Indicadores de rendimiento, Colección electrónica, Servicios electrónicos, Biblioteca digital.

Title: Statistics and performance indicators for electronic library collections and services: a case study of electronic journals

Abstract: The use of statistics and performance indicators for evaluating electronic library collections and services is analysed. Major projects of recent years that have defined statistics and performance indicators are examined, with special attention paid to three of them: COUNTER, E-metrics and ISO. The statistics currently offered by four electronic journal publishers (American Chemical Society, Emerald, Kluwer, and Wiley) as well as by one service (Scitation Usage Statistics) are analysed. Results show a certain degree of consensus on a basic set of statistics and indicators despite the diversity of existing projects and the heterogeneity of data offered by the publishers.

Keywords: Statistics, Performance indicators, Electronic collections, Electronic services, Digital library.

Borrego, Ángel; Urbano, Cristóbal. "Estadísticas e indicadores de rendimiento de colecciones y servicios bibliotecarios de carácter electrónico: estudio de caso de las revistas electrónicas”. En: El profesional de la información, 2005, enero-febrero, v. 14, n. 2, pp. 30-38.

\section{Artículo}

Ángel Borrego, profesor de la Facultat de Biblioteconomia i Documentació de la Participa en el proyecto «Dadición para la mebliotecario: desarrollo de un mara bibliotecas unifaly financiado por el Plan Nacional I+D+i del Ministerio de Educación y Ciencia.

Cristóbal Urbano, profesor titular de la Universitat de Barcelona, responsable del Grupo de Investigación $\pi$-EMEI (Plataforma de Integración de Estudios Métricos y Estadísticos de Información) del Departamento de Biblioteconomía y Documentación de la UB.

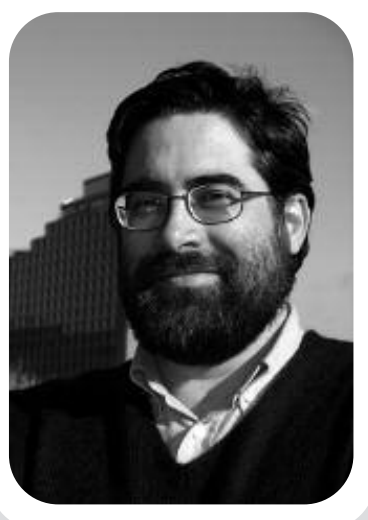

\section{Introducción}

\subsection{La medición del rendimiento}

Es una herramienta integrada en los procesos de evaluación que consiste en la recogida y análisis de datos objetivos descriptivos del funcionamiento de la biblioteca. Poll y Boekhorst (1998, p. 20) la definen como la recogida de datos estadísticos y de otro tipo que describan la actividad de la biblioteca, así como el análisis de estos datos con la finalidad de evaluar su rendimiento. El rendimiento sería el grado de cumplimiento de los objetivos por parte de la biblioteca; un indicador de rendimiento es una proposición cuantificada (que relaciona un resultado con los recursos invertidos para obtenerlo) utilizada para determinar el grado de consecución de esos objetivos. 
La introducción en las bibliotecas de servicios y contenidos de carácter electrónico ha obligado a emplear nuevos métodos de cuantificación y unidades de medición del uso de estos servicios y colecciones. Asimismo, las fuentes de obtención de los datos estadísticos han variado respecto a las empleadas en el caso de recursos y servicios de información impresos.

Una de las nuevas técnicas que permite recoger datos sobre el uso de productos y servicios de información electrónicos - visitas, visitantes, páginas visitadas, hits, duración de las sesiones, fechas y horas de consulta, secuencias de navegación, páginas de entrada y salida, procedencia geográfica de los usuarios, navegador que se utiliza, sistema operativo, etc.- es el análisis transaccional. Es muy útil para determinar cuáles son los recursos que se utilizan y con qué frecuencia, así como para formular hipótesis sobre el comportamiento de los usuarios. No obstante, para saber por qué (o por qué no) se utiliza un producto o servicio se requiere entrar en comunicación directa con los usuarios a través de encuestas, entrevistas, grupos de discusión, etc.

\section{«El rendimiento es el grado de cumplimiento de los objetivos y un indicador de rendimiento es una proposición cuantifica- da utilizada para determinar el grado de consecución de esos objetivos»}

Además, en el entorno digital es necesario encontrar nuevas medidas del uso de los servicios y colecciones como el tiempo de conexión, el número de sesiones, la cantidad de sesiones simultáneas, el número de hits, de usuarios activos, etc. Algunas de estas medidas pueden verse afectadas por variables incontroladas (el tiempo de conexión puede depender del tráfico en la Red, el número de hits varía si se utiliza una $c a$ ché, etc.). Brophy y Wynne (1997, p. 36) apuestan por utilizar el número de sesiones como medida básica del uso, ya que presenta diversas ventajas: es independiente del tiempo, permite efectuar desgloses (por facultad, departamento, etc.), da una idea aproximada del uso comparado de los servicios, permite construir series temporales, facilita la comparación entre instituciones, etc.

Podemos definir una sesión como una conexión a un servicio electrónico resuelta con éxito, es decir, que ha funcionado, independientemente del tiempo necesario para realizarla. Comienza cuando alguien se conecta a un servicio, por ejemplo una base de datos, y finaliza cuando se desconecta, ya sea intencionada- mente o porque supera el tiempo máximo de inactividad permitido.

Por otra parte, es necesario hacer notar que las fuentes de datos para la medición del uso de productos y servicios electrónicos de información son dos: por un lado, hay datos generados localmente (por ejemplo las consultas a un cd-rom local) y, además, datos generados por el uso local de una fuente remota (las consultas locales a un cd-rom alojado en un servidor remoto). En este último caso las estadísticas de uso han de ser suministradas por el editor o distribuidor del recurso.

A diferencia de lo que pasaba tradicionalmente, las bibliotecas han dejado de ser autosuficientes en el proceso de recogida de datos del uso de sus colecciones y, en el caso de los recursos electrónicos, dependen de una segunda instancia: los editores. A pesar de que la biblioteca puede obtener información sobre cómo navegan los usuarios a través de su web o sobre cuáles son los recursos más solicitados en su catálogo, una vez que el usuario se conecta a un recurso remoto se pierde su pista. En ese momento depende del proveedor para obtener estadísticas sobre el uso que se hace de los productos que la institución tiene contratados. Este hecho ocasiona algunos problemas desde el momento en que diferentes editores no mantienen unas prácticas uniformes en lo referente a la recogida y suministro a las bibliotecas de los datos de uso de los productos electrónicos. Por una parte, los diversos editores suministran datos diferentes que, por tanto, no son comparables y por otro lado en ocasiones no queda claro qué es lo que se está ofreciendo debido a la falta de definiciones que aclaren el significado concreto de las informaciones ofrecidas. A menudo, los proveedores que ofrecen estadísticas de uso utilizan una terminología idéntica o muy similar (términos como "búsquedas", "descargas", "sesiones" o "visualizaciones" son muy habituales) para referirse a realidades diferentes. La coexistencia de definiciones, formatos y períodos de recogida poco o nada normalizados dificulta enormemente, hoy por hoy, el aprovechamiento real de los datos.

No obstante, el hecho de que sean los editores los que recogen los datos de uso también comporta alguna ventaja. Son ellos quienes se hacen cargo de los gastos que esta actividad produce, a pesar de que algunos ya se quejan de que ofrecer estas estadísticas tiene un coste adicional que difícilmente mantendrán sin obtener a cambio una compensación económica. Por otro lado, aparte del coste económico que puede generar, algunos también se muestran poco inclinados a ofrecer estadísticas de uso a los bibliotecarios por temor a que éstos las utilicen para justificar la cancelación de productos poco utilizados. Finalmente, dado que el núme- 
ro de grandes editores es relativamente reducido, debería ser factible que en un plazo breve de tiempo se llegara a la adopción de un estándar relativo a la recogida, tratamiento y difusión de las estadísticas de uso de productos electrónicos.

De entre el amplio abanico de indicadores existentes, desarrollados en numerosos proyectos e iniciativas que comentaremos más adelante, cada biblioteca ha de optar por aquellos que considere más idóneos en función de sus características, los objetivos del proceso de evaluación en el que se encuentra involucrada o sus limitaciones de personal, presupuestarias, temporales o de otro tipo, entre otros factores.

Por otro lado, es necesario mantener las estadísticas e indicadores analizados en unos mínimos. Los procesos de recogida y análisis son costosos y los recursos empleados en su obtención deben estar justificados por el interés y uso posterior de los datos. Por esta razón, antes de decidir recoger alguna nueva estadística o elaborar un nuevo indicador es interesante plantearse un cuestionario de evaluación de la necesidad y adecuación de las estadísticas como el propuesto por Ryan, McClure y Bertot (2001, p. 116).

A continuación analizaremos los principales proyectos de definición de estadísticas e indicadores para la evaluación del rendimiento de productos y servicios de información electrónicos desarrollados durante los últimos años. Prestaremos especial atención a tres:

-Counter (Counting Online Usage of Networked Electronic Resources), por estar convirtiéndose en una suerte de estándar,

-E-metrics, por su importancia entre las bibliotecas universitarias norteamericanas al estar desarrollado desde la ARL (Association of Research Libraries) y,

- los proyectos de ISO por tratarse de la institución normalizadora que más nos afecta en nuestro ámbito geográfico.

Finalmente, analizaremos las estadísticas que actualmente procuran cuatro grandes editores de revistas electrónicas (American Chemical Society, Emerald, Kluwer y Wiley) y un servicio (Scitation Usage Statistics) que aglutina datos de seis editores de revistas de física.

\section{Proyectos de definición de estadísticas e indicadores de rendimiento de productos y servicios de información electrónicos}

\subsection{Precedentes}

Uno de los primeros fue el llevado a cabo por Brophy y Wynne (1997) que propusieron un conjun- to de indicadores divididos en tres categorías correspondientes a los tres niveles de gestión bibliotecaria que los autores consideraban: gestión operativa (la organización y control, día a día, de los recursos y servicios), planificación y evaluación. En el último de los tres, el informe seguía el esquema de indicadores propuesto en La biblioteca universitaria eficaz (1999; edición original de 1995), modificando algunos de los ya existentes para incluir aspectos relativos a la provisión de información electrónica e incorporando otros dirigidos específicamente a la evaluación de la biblioteca electrónica.

El proyecto Equinox (2000), financiado por la Comisión Europea dentro del programa Telematics for $\mathrm{li}$ braries, se desarrolló entre 1998 y 2000. Su objetivo primordial era determinar una serie de indicadores para medir el rendimiento de los nuevos servicios electrónicos que ofrecían las bibliotecas y que vendrían a completar los ofrecidos en la norma ISO 11620 (ISO, 1998). Como resultado se desarrolló un listado de 14 indicadores y en su sede web se informa con detalle del objetivo, definición, método de cálculo e interpretación de cada indicador.

http://equinox.dcu.ie

Roswitha Poll, una de las coautoras del manual de la IFLA para la medición del rendimiento en bibliotecas universitarias (Poll; Boekhorst, 1998; edición original de 1996) basándose en la lista de indicadores propuesta por el proyecto Equinox y por el grupo de trabajo de $I S O$ encargado de la elaboración del informe técnico 20983 (ISO, 2003b), elaboró un listado de 15 indicadores agrupados en seis categorías (Poll, 2001).

\section{«No obstante, para saber por qué (o por qué no) se utiliza un producto o servicio se requiere entrar en comunicación directa con los usuarios a través de encuestas»}

Según esta autora (Poll, 2001, p. 245) para la evaluación de servicios electrónicos es necesario recoger datos sobre el tamaño de la colección electrónica (revistas, bases de datos, documentos individuales, etc.), el uso de los servicios (colección electrónica, opac, página web de la biblioteca, etc.) y los gastos en servicios electrónicos (colección, equipamientos, etc.). Para la medición del uso, Poll (2001, p. 247) recomienda restringir la contabilidad a tres variables: sesiones, sesiones rechazadas y documentos vistos. Al mismo tiempo, sería necesario hacer un desglose de estas variables en función de la procedencia de los usuarios 
(dentro de la biblioteca, fuera de ella pero dentro de la institución, o fuera de la institución).

Bertot, McClure y Ryan publicaron en 2001 un manual en el que también establecían un conjunto de indicadores para medir el rendimiento de servicios electrónicos ofrecidos en bibliotecas públicas. Los autores recomendaban recabar un conjunto de 13 estadísticas (pp. 7-8) de las que ofrecían definiciones, procedimientos de recogida, informaciones sobre su interpretación, etc. Además, se incluían diversos capítulos sobre el desarrollo de cuestionarios y grupos de discusión para obtener datos en relación a la opinión de los usuarios, instrucciones para el proceso de recogida de datos y construcción de indicadores, aspectos a considerar en la selección de las medidas a aplicar en cada biblioteca, así como un listado de estadísticas que se deberían analizar con más detalle antes de recomendar su recogida (pp. 81-88).

En diciembre de 2001, Icolc (International Coalition of Library Consortia) publicó unas pautas para medir estadísticamente el uso de recursos de información basados en el web (Icolc, 2001). El documento definía un conjunto de elementos mínimos que los proveedores de información tenían que suministrar a los administradores de consorcios.

\section{«La coexistencia de definicio- nes, formatos y períodos de re- cogida poco o nada normaliza- dos dificulta enormemente, hoy por hoy, el aprovechamiento re- al de los datos»}

Por otra parte, la University of Central England ha venido trabajando durante 2004 en el proyecto Libraries: outcomes and measures (Evidence, 2004), en cuyo marco se han definido un conjunto de 16 medidas agrupadas en tres categorías: colección, uso y costes. Paralelamente, el proyecto eValued (eValued, 2004) ha desarrollado un conjunto de herramientas para la medición del rendimiento de servicios electrónicos que sea aplicable en las bibliotecas universitarias británicas. Este "archivo de herramientas", como ellos lo denominan, incluye directrices y ejemplos de preparación de cuestionarios, entrevistas, grupos de discusión, etc. reunidos en categorías temáticas y en función del tipo de destinatarios (estudiantes, profesores, investigadores, etc.).

Finalmente, cabe destacar que en octubre de 2004, NISO publicó la tercera revisión de la norma Z39.7 sobre medición del rendimiento bibliotecario bajo el título Information services and use: metrics \& statistics for libraries and information providers-data dictio-

\section{Nivel I}

- Informe de revistas I: número de descargas de artículos a texto completo realizadas con éxito por mes y título.

- Informe de revistas Ib: número de descargas de artículos a texto completo realizadas con éxito en formato html y pdf.

- Informe de revistas 2: sesiones rechazadas por mes y título.

- Informe de bases de datos I: total de búsquedas y sesiones por mes y base de datos.

- Informe de bases de datos 2: sesiones rechazadas por mes y base de datos.

- Informe de bases de datos 3: total de búsquedas y sesiones por mes y servicio.

\section{Nivel 2}

- Informe de revistas 3: número de peticiones de ítems con éxito y sesiones rechazadas por mes, título y tipo de página.

- Informe de revistas 4: total de búsquedas realizadas por mes y servicio.

Tabla 1. Medidas definidas en el proyecto Counter (Counter, 2004)

nary ANSI/NISO Z39.7-200X (NISO, 2004). La norma incluye medidas sobre información electrónica, destacadas en al documento con la expresión "Emetrics", en tres de sus secciones: colecciones, finanzas y servicios. Además de las definiciones de las medidas incluye, entre sus cinco apéndices, tres de interés sobre el tema que nos ocupa: B) Measuring the use of electronic library services; C) Measuring public library networked services: preparing your library to collect network statistics, elaborado por John Carlo Bertot; y E) National and international efforts regarding electronic metrics.

\subsection{Counter}

Se puso en marcha en marzo de 2002 como una iniciativa internacional (que aglutina a bibliotecarios, editores y proveedores) encaminada a facilitar el registro e intercambio de estadísticas de uso de información en línea. En diciembre de 2002 se publicó un $C o$ de of practice (Counter, 2002) que ofrecía una guía sobre los datos que se han de medir, definiciones, contenido y formato de los informes en los que los editores han de comunicar estos datos, e información sobre el proceso de análisis; en abril de 2004 se dio a conocer su segunda versión (Counter, 2004). Recientemente, a finales de septiembre de 2004, se ha cerrado el período de recepción de alegaciones a esta nueva versión y está previsto que la definitiva se publique en enero de 2005 para entrar en vigor en enero de 2006. 
Tanto la primera como la segunda versión del Code of practice definen dos niveles de informes que tienen que ofrecer los proveedores. El primero indica los datos mínimos que un proveedor ha de procurar para alcanzar la categoría de "Countercompliant", mientras que el segundo establece una serie de elementos aún no obligatorios para obtener esta acreditación, pero que lo serán en el futuro. En la sede web del proyecto se puede consultar el listado de editores que cumplen el código y en la tabla 1 se presentan los dos niveles de medidas definidos en la ultima versión del Code of practice. http://www.projectcounter.org

En esta primera fase, Counter se ha centrado en las revistas y bases de datos, no sólo porque se trata de los materiales para los que se consignan la mayor parte de los presupuestos bibliotecarios, sino porque son los que llevan más tiempo disponibles y, por tanto, ya existe un conjunto relativamente amplio de definiciones aceptadas. Otros materiales, como los libros electrónicos, deberán incorporarse en ediciones posteriores.

\subsection{E-metrics (ARL)}

La ARL cuenta con una larga tradición en la obtención de estadísticas bibliotecarias, actividad en la que destaca por la publicación anual, desde 1962, de los datos correspondientes a los miembros de la asociación. Ante el reto que suponía la recogida de estadísticas sobre productos y servicios electrónicos llevó a cabo, entre mayo de 2000 y diciembre de 2001, el proyecto E-metrics.

\section{http://www.arl.org/stats/newmeas/emetrics/}

Se desarrolló en tres fases (Shim, et al., 2000, 2001a y 2001b): en la primera se realizó un inventario de las prácticas de recogida de estadísticas sobre servicios electrónicos por parte de las bibliotecas de la $A R L$ y se establecieron contactos con editores; a continuación se identificaron y probaron un conjunto de medidas para la evaluación del rendimiento; y, finalmente, se intentaron institucionalizar los procesos de recogida y cálculo de las estadísticas e indicadores. Como resultado de la segunda fase del estudio se iden-

\section{- Coste de los libros electrónicos. \\ - Gastos de la biblioteca en herra- mientas bibliográficas, redes y con- sorcios. \\ - Gastos externos en herramientas bibliográficas, redes y consorcios. \\ Actividades de digitalización de la bi- blioteca: \\ - Tamaño de la colección digital de la biblioteca. \\ - Uso de la colección digital de la bi- blioteca. \\ - Coste de la construcción y mante- nimiento de la colección digital de la biblioteca. \\ Indicadores de rendimiento \\ - Porcentaje de las consultas de refe- rencia electrónicas respecto al total de consultas de referencia. \\ - Porcentaje de las visitas virtuales a la biblioteca respecto al total de vi- sitas a la biblioteca. \\ - Porcentaje de libros electrónicos respecto al total de monografías. \\ Coste de las fuentes de referencia electrónicas.}

tificaron un conjunto de 16 medidas estadísticas agrupadas en 4 categorías, así como 3 indicadores de rendimiento que aparecen en la tabla 2. En realidad, aunque el documento de la $A R L$ los califica así, los indicadores no son propiamente de rendimiento, sino ratios de crecimiento de los servicios y colecciones electrónicos.

\subsection{International Organization for Standardiza- tion (ISO)}

En 1974 se publicó la ISO 2789: 1974, con ediciones posteriores de 1991 y 2003, sobre la recogida de estadísticas bibliotecarias. Esta última edición, la de 2003 (ISO, 2003a) incluye definiciones de datos relacionados con la prestación de servicios electrónicos y un anexo específico sobre el tema: Measuring the use of electronic library services (pp. 35-43). De acuerdo con la norma, las bibliotecas ofrecen en la actualidad los siete servicios electrónicos representados en la figura 1.

La norma define cuatro datos que se tendrían que recoger para medir el uso de la colección electrónica (número de sesiones, documentos descargados, registros descargados y cantidad de visitas virtuales) así co- 


\section{(5) \\ baratz}

\section{En primera línea en}

Sistemas de Información y Gestión del Conocimiento

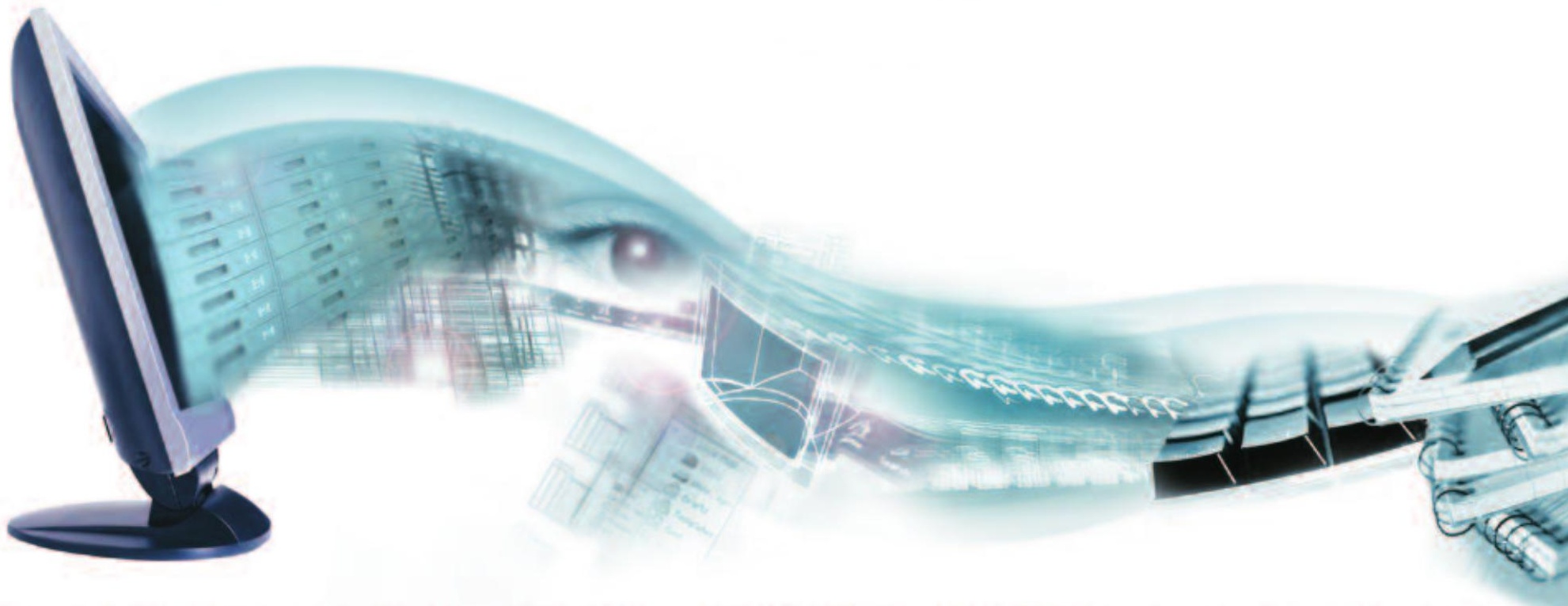

Raimundo Fernández Villaverde, 2828003 Madrid (España) Teléfono +34914560360 - Fax +34915330958 www.baratz.es - E.mail: informa@baratz.es

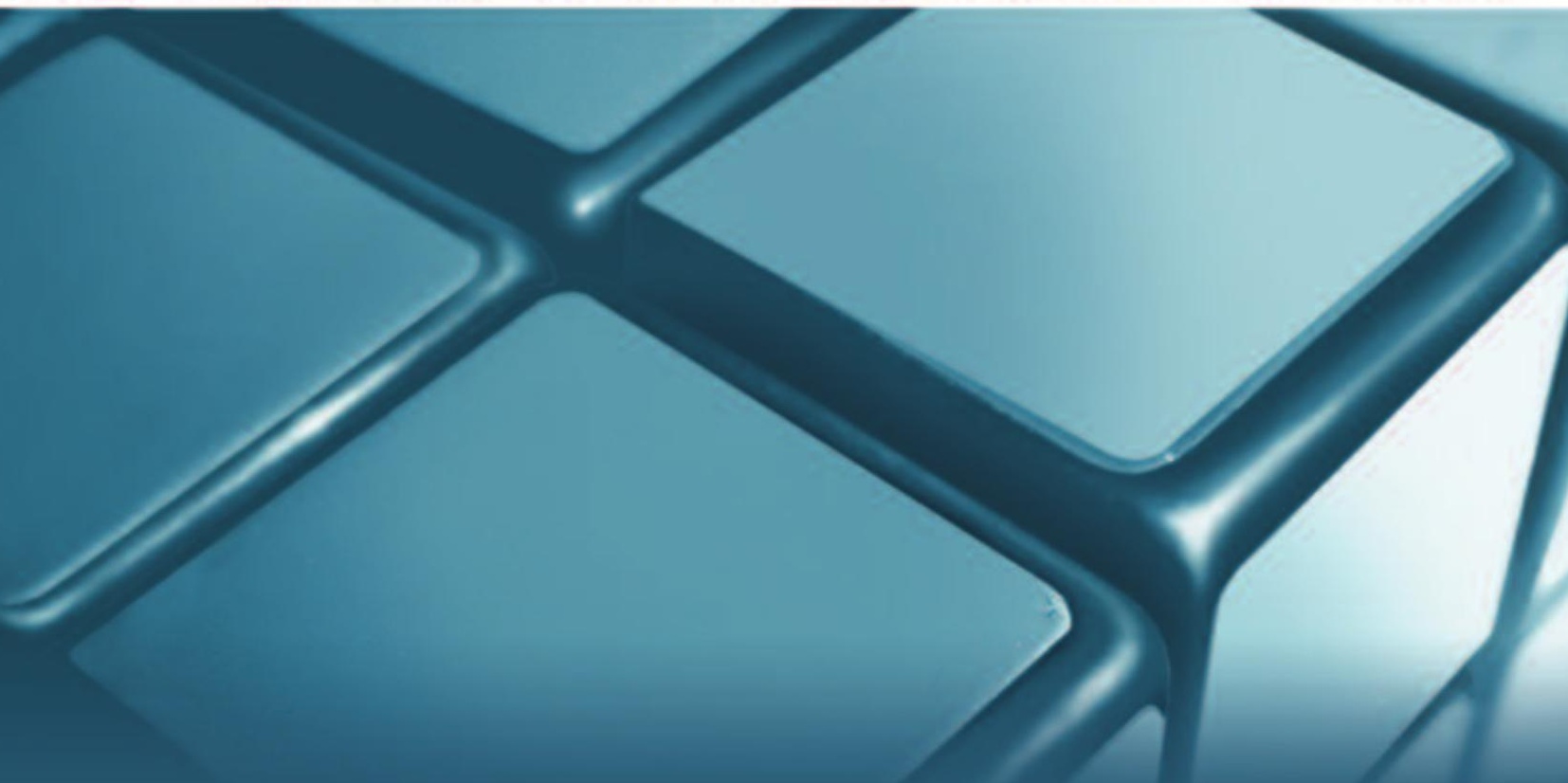

(2) Soluciones para bibliotecas:

Absys, absysNET, Absys express

(2) Gestión documental y del conocimiento: BKM, Baratz Windows, BRSCGI

(2) Soluciones para archivos: Albalá

(9) Servicios de Catalogación Retrospectiva

(2) Edición en CD-Rom

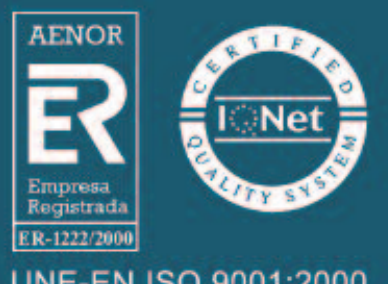




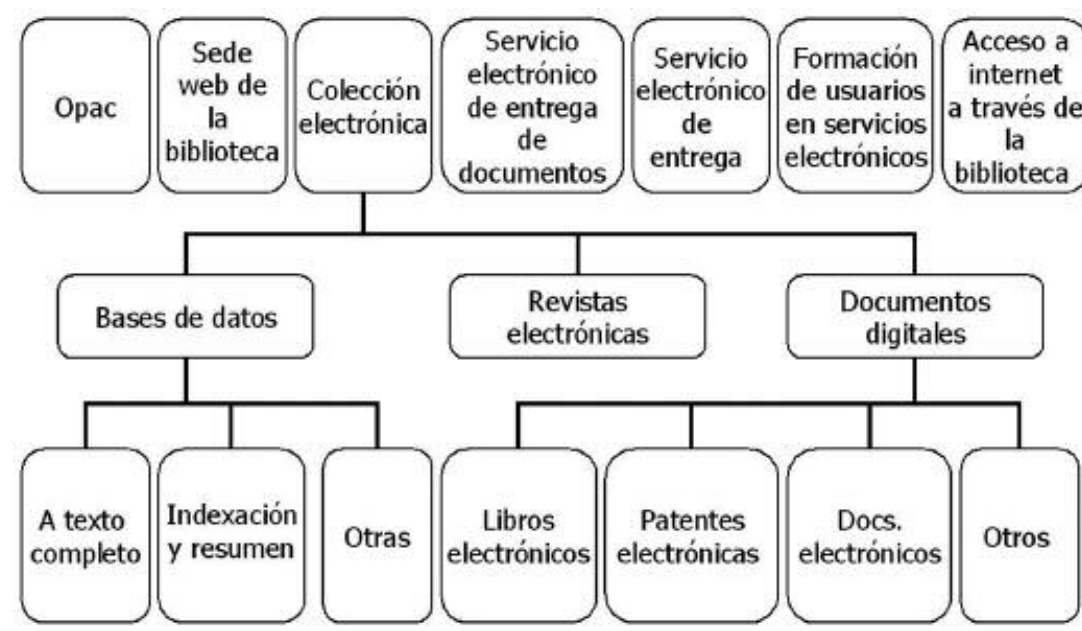

Figura 1. Servicios electrónicos relevantes (ISO, 2003a, p. 38)

\section{Estadísticas ofrecidas por una muestra de editores de revistas electrónicas}

En este último apartado analizaremos las estadísticas que actualmente, en octubre de 2004, ofrecen cinco grandes editores de revistas científicas: American Chemical Society, Emerald, Kluwer, Scitation Usage Statistics Service y Wiley. Como ejemplo del tipo de análisis que se pueden realizar a partir de estas estadísticas se puede consultar el informe y artículo realizados sobre el consumo de cuatro paquetes de revistas electrónicas por el Consorci de Bibliotemo cuatro elementos adicionales que se deberían reunir cuando fuera posible y apropiado (duración de la sesión, número de sesiones rechazadas, número de búsquedas y sesiones de internet). En cada caso deberían diferenciarse tres posibles localizaciones del usuario: dentro de la biblioteca, fuera de ella pero dentro de la institución, y fuera de la institución.

Por otra parte, en 1998 se publicó la ISO 11620 dirigida a la promoción del uso de indicadores de rendimiento bibliotecario. La norma definía un conjunto de 29 indicadores y, para cada uno de ellos, definía su objetivo, campo de aplicación, definición, método de cálculo e interpretación y factores que le afectan. Recientemente, en 2003, se publicó una extensión de esta norma -Amendment 1/2003: Additional performance indicators for libraries (ISO, 2003b) que incluía 5 nuevos indicadores de rendimiento para llegar a un total de 34. También en 2003, ISO publicó el informe técnico 20983 (ISO, 2003c) sobre indicadores de rendimiento de servicios electrónicos que presentaba definiciones y descripciones de una selección de indicadores siguiendo el formato de la ISO 11620. De hecho, el objetivo es que, cuando estos indicadores estén más trabajados, se incorporen a una futura revisión de la ISO 11620. El informe ofrece la lista de indicadores de rendimiento para servicios electrónicos de la tabla 3.

\section{Servicios públicos}

I.I. General.

- Porcentaje de la población a la que llegan los servicios electrónicos.

I.2. Provisión de servicios bibliotecarios electrónicos.

- Porcentaje de gasto en provisión de información dedicado a la colección electrónica.

I.3. Recuperación de documentos.

- Número de documentos descargados por sesión.

- Coste por sesión en bases de datos.

- Coste por documento descargado.

- Porcentaje de sesiones rechazadas.

- Porcentaje de sesiones remotas en el opac.

- Visitas virtuales como porcentaje del total de visitas.

I.4. Servicio de referencia.

- Porcentaje de peticiones de información enviadas electrónicamente.

\section{I.5. Formación de usuarios.}

- Número de asistencias de usuarios a sesiones de formación en servicios electrónicos per capita.

I.6. Infraestructuras.

- Horas de estaciones de trabajo disponibles per capita.

- Población por estación de trabajo de acceso público.

- Tasa de uso de las estaciones de trabajo.

\section{Disponibilidad y uso de los recursos humanos}

2.I. Formación de la plantilla.

- Número de asistencias a sesiones de formación en nuevas tecnologías por miembro de la plantilla.

2.2. Utilización del personal.

- Porcentaje de la plantilla que ofrece y desarrolla servicios electrónicos.
Tabla 3. Indicadores de servicios electrónicos de ISO (ISO/TR 20983: 2003, pp. 10-11) 
informes mensuales de consultas por dirección Ip, con lo que se puede saber cuántas descargas de artículos y resúmenes se han hecho de cada título desde cada dirección.

http://pubs.acs.org

\subsection{Emerald}

Lo primero que cabe destacar es que ofrece una completa guía para el usuario (Emerald, 2004) en la que se define y se explica el funcionamiento del portal de estadísticas. Ofrece datos de sumarios y resúmenes vistos, descargas de artículos, descargas de artículos rechazadas y sesiones de usuarios. Cada uno de estos cinco datos se puede consultar por título y mes; se visualizan en forma de tabla o gráfica, facilitándose su descarga para poder trabajarlos con una hoja de cálculo. Adicionalmente es posible solicitar por correo electrónico los informes definidos en el proyecto Counter. http://www.emeraldinsight.com

\subsection{Kluwer}

Es uno de los editores que ofrecen estadísticas más completas. Por desgracia, la utilidad de estos datos es limitada ya que no hay un manual con definiciones de los términos, por lo que en ocasiones resulta difícil saber con exactitud a qué se refiere cada uno. Suministra información sobre la navegación que hacen los usuarios (indicando cuáles son las páginas de entrada y salida del web del editor), dominios desde los que se accede a la información, búsquedas (con indicación de los términos buscados y si la consulta ha tenido éxito o no), artículos y resúmenes más consultados, etc. En lo que se refiere estrictamente al consumo de las revistas, ofrece los informes de revistas 1 (número de descargas de artículos a texto completo realizadas con éxito por mes y título) y 3 (número de peticiones de ítems con éxito y rechazos por mes, título y tipo de página) de Counter, las páginas vistas por título y mes, así como los accesos denegados por título y mes.

http://journals.kluweronline.com/

\subsection{Scitation Usage Statistics Service}

Este servicio incluye datos estadísticos de uso de seis editores de revistas de física - American Institute of Physics; American Physical Society; American Association of Physics Teachers; American Society of Civil Engineers; ASME Internacional; y SPIE (The International Society for Optical Engineering)—. Para cada título se ofrecen datos de artículos descargados, resúmenes consultados, búsquedas realizadas, sumarios vistos y visitas a la página inicial de la revista. http://scitation.aip.org/jhtml/inst/serviceinfo.jsp

\subsection{Wiley}

Ofrece datos mensuales y acumulaciones anuales de la actividad de los usuarios: sesiones, hits, búsque- das, sumarios vistos, accesos denegados y descargas de artículos (desglosadas en html y pdf) y en función de si son resultado de una búsqueda o de una actividad de browsing. Asimismo, suministra datos de los 10 títulos más consultados, los 10 que los usuarios intentan consultar con más asiduidad sin estar subscritos, y el informe de revistas 1 de Counter (número de descargas de artículos a texto completo realizadas con éxito por mes y título).

http://www3.interscience.wiley.com

\section{Conclusiones}

La revisión de la bibliografía muestra el interés prestado durante los últimos años al desarrollo de estadísticas e indicadores de rendimiento que se integren en los procesos de evaluación bibliotecaria. En líneas generales, el análisis se centra en la cuantificación de los recursos invertidos, los procesos realizados y los resultados obtenidos. Sin embargo, el impacto (el efecto de los resultados sobre el entorno como, por ejemplo en una biblioteca universitaria, el grado en que su uso afecta al aprendizaje de los alumnos o a la producción científica de la institución) resulta más difícil, o quizá imposible, de medir. Por esta razón la evaluación del rendimiento se limita, a menudo, a la recogida de estadísticas cuantitativas sobre el funcionamiento de la biblioteca.

\section{«En 2003, ISO publicó el infor- me técnico 20983 sobre indica- dores de rendimiento de servi- cios electrónicos que presenta- ba definiciones y descripciones de una selección de indicado- res siguiendo el formato de la ISO 11620»}

$\mathrm{Al}$ analizar las características de los diversos proyectos de determinación de estadísticas e indicadores de rendimiento para productos y servicios electrónicos se observa, a pesar de la disparidad de las propuestas, un cierto grado de consenso en torno a una serie de variables cuya medición resulta inexcusable. Así, se podrían establecer cinco grandes categorías de productos y servicios a medir: colección electrónica, formación de usuarios, opac, sede web y servicio de referencia. La medición del uso de cada uno de estos productos y servicios requerirá de unidades diferentes según sus características. Así, en el caso de la colección electrónica será necesario cuantificar su tamaño (número de bases de datos, documentos digitales y revistas electrónicas disponibles) y su uso a través de diferentes medidas (búsquedas, documentos consultados, sesiones y sesiones rechazadas). 
Estas medidas deberán desglosarse en función de la procedencia de los usuarios: dentro de la biblioteca, fuera de la biblioteca pero dentro de la institución, o fuera de la institución. En el caso de la formación de usuarios deberá cuantificarse el número de horas y asistentes a las sesiones; el uso del opac y la sede web se medirá a través del número de visitas, páginas vistas y búsquedas; y el servicio de referencia electrónico se evaluará a partir del número de consultas realizadas y contestadas electrónicamente. Finalmente, deberán cuantificarse los recursos invertidos en la biblioteca electrónica, ya sea en la compra de documentos (adquisición de materiales o contratación del acceso a recursos remotos), habilitación de infraestructuras (hardware y software) y contratación y formación de personal.

\section{«Si hay un modelo que parece ir camino de convertirse en un estándar es el de Counter»}

Por último, en cuanto a las estadísticas que actualmente ofrecen los editores cabe destacar la escasa homogeneidad en los datos ofrecidos, aunque si hay un modelo que parece ir camino de convertirse en un estándar es el de Counter. En todo caso, a la espera de que haya una mayor uniformidad, es necesario que los editores ofrezcan definiciones de los términos que utilizan para facilitar un mejor aprovechamiento de los datos suministrados. Por otro lado, resulta necesario que las bibliotecas institucionalicen sus propios procesos de recogida de datos allí donde puedan obtener información sobre el comportamiento de sus usuarios. Estos datos serán útiles para completar las estadísticas ofrecidas por los editores y contrastar su fiabilidad.

\section{Referencias}

Bertot, John Carlo; McClure, Charles R.; Ryan, Joe. Statistics and performance measures for public library networked services. Chicago; London: American Library Association, 2001. Isbn 0-8389-0796-2.

La biblioteca universitaria eficaz: directrices para la evaluación del rendimiento en bibliotecas universitarias: informe consultivo para Hefce, Shefc, Hefcw y DENI. Cádiz: Servicio de Publicaciones de la Universidad de Cádiz, 1999. Isbn 84-7786-609-0.

Brophy, Peter; Wynne, Peter M. Management information systems and performance measurement for the digital library: eLib supporting study (MIEL 2). June 1997. Consultado en: 16-11-04.

http://www.ukoln.ac.uk/services/elib/papers/supporting/pdf/mis.pdf

Counter. The Counter code of practice. Release 1: December 2002. Consultado en: 16-11-04.

http://www.projectcounter.org/codeofpractice.pdf

Counter. The Counter code of practice. Release 2: April 2004. Consultado en: 16-11-04.

http://www.projectcounter.org/code_of_practice_release_2.pdf

Emerald Usage Statistics: User Guide v1.3. Consultado en: 16-11-04. http://www.emeraldinsight.com/stats/eusg.doc

Equinox: library performance measurement and quality management system. Updated November 2000. Consultado en: 16-11-04.

http://equinox.dcu.ie
Evidence base: research \& evaluation. Consultado en: 16-11-04. http://www.ebase.uce.ac.uk/emeasures/

Evalued: an evaluation toolkit for e-library developments. Consultado en: 16-11-04.

http://www.evalued.uce.ac.uk.

Icolc. Guidelines for statistical measures of usage of web-based information resources. Update: December 2001. Consultado en: 16-11-04. http://www.library.yale.edu/consortia/2001webstats.htm

Hay traducción al catalán: Pautes per mesurar estadísticament l'ús de recursos d'informació basats en web. Consultado en: 16-11-04. http://www.cbuc.es/5digital/Tur2def.pdf

ISO. ISO 11620: 1998. Information and documentation: library performance indicators. Geneva: ISO, 1998.

ISO. ISO 2789: 2003. Information and documentation: international library statistics. Geneva: ISO, 2003.

ISO. ISO 11620: 1998. Amd. 1: 2003. Additional performance indicators for libraries. Geneva: ISO, $2003 \mathrm{~b}$.

ISO. ISO/TR 20983. Information and documentation: performance indicators for electronic library services. Geneva: ISO, 2003c.

NISO. Information services and use: metrics \& statistics for libraries and information providers-data dictionary ANSI/NISO Z39.7-200X. Consultado en: 07-10-04.

http://www.niso.org/emetrics/

Poll, Roswitha; Boekhorst, Peter Te. Medición de la calidad: directrices internacionales para la medición del rendimiento en las bibliotecas universitarias. Madrid: Anabad, 1998. Isbn 84-88716-27-3.

Poll, Roswitha. "Performance indicators for the digital library". En: $L i$ ber quarterly, 2001, v. 11, n. 3, pp. 244-258.

Ryan, Joe; McClure, Charles R.; Bertot, John Carlo. "Choosing measures to evaluate networked information resources and services: selected issues". En: Evaluating networked information services: techniques, policy and issues. Edited by Charles McClure and John Carlo Bertot. Medford: Information Today, 2001, pp. 111-135. Isbn 1-57387-118-4.

Shim, Wonsik “Jeff”; McClure, Charles R.; Bertot, John Carlo. ARL e-metrics project: developing statistics and performance measures to describe electronic information services and resources for ARL libraries. Phase one report. November 7, 2000. Consultado en: 07-10-04.

http://www.arl.org/stats/newmeas/emetrics/phaseone.pdf

Shim, Wonsik “Jeff”; McClure, Charles R.; Fraser, Bruce T.; Bertot, John Carlo; Dagli, Arif; Leahy, Emily H. Measures and statistics for research libraries networked services: procedures and issues. ARL metrics phase II report. October 2001a. Consultado en: 07-10-04.

http://www.arl.org/stats/newmeas/emetrics/phasetwo.pdf

Shim, Wonsik “Jeff”; McClure, Charles R.; Fraser, Bruce T.; Bertot John Carlo. Data collection manual for academic and research library network statistics and performance measures. December 2001b. Consultado en: 07-10-04.

http://www.arl.org/stats/newmeas/emetrics/phase3/ARL_Emetrics_Data_ Collection_Manual.pdf

Urbano, Cristóbal; Borrego, Ángel; Cosculluela, Antoni; Cantos, Carme. Ús de revistes electròniques de compra consorciada del CBUC (2000-2003). Març 2004. Consultado en: 07-10-04.

http://www.cbuc.es/5digital/Informe_revistes_CBUC_amb_grafics.pdf

Urbano, Cristóbal; Anglada, Lluís; Borrego, Ángel; Cantos, Carme; Cosculluela, Antonio; Comellas, Núria. "The use of consortially purchased electronic journals by the CBUC (2000-2003)". En: D-lib magazine, 2004, v. 10, n. 6. Consultado en: 07-10-04.

http://www.dlib.org/dlib/june04/anglada/06anglada.html

Ángel Borrego, Cristóbal Urbano, Universitat de Barcelona, Facultat de Biblioteconomia i Documentació, Melcior de Palau, 140, 08014 Barcelona.

borrego@ub.edu

urbano@ub.edu 